\title{
Genome-wide copy number variant analysis reveals variants associated with 10 diverse production traits in Holstein cattle
}

\author{
Yang Zhou ${ }^{1,2,3}$, Erin E. Connor ${ }^{1} \mathbb{D}$, George R. Wiggans ${ }^{1}$, Yongfang Lư ${ }^{4}$, Robert J. Tempelman ${ }^{4}$, Steven G. Schroeder ${ }^{1}$, \\ Hong Chen ${ }^{2}$ and George E. Liu ${ }^{1 *}$
}

\begin{abstract}
Background: Copy number variation (CNV) is an important type of genetic variation contributing to phenotypic differences among mammals and may serve as an alternative molecular marker to single nucleotide polymorphism (SNP) for genome-wide association study (GWAS). Recently, GWAS analysis using CNV has been applied in livestock, although few studies have focused on Holstein cattle.

Results: We describe 191 CNV detected using intensity data from over 700,000 SNP genotypes generated with the BovineHD Genotyping BeadChip (Illumina, San Diego, CA) in 528 Holstein cows. The CNV were used for GWAS analysis of 10 important production traits of 473 cattle related to feed intake, milk quality, and female fertility, as well as 2 composite traits of net merit and productive life. In total, we detected 57 CNV associated $(P<0.05$ after false discovery rate correction) with at least one of the 10 phenotypes. Focusing on feed efficiency and intake-related phenotypes of residual feed intake and dry matter intake, we detected a single CNV associated with both traits which overlaps a predicted olfactory receptor gene OR2A2 (LOC787786). Additionally, 2 CNV within the RXFP4 (relaxin/insulin like family peptide receptor 4) and 2 additional olfactory receptor gene regions, respectively, were associated with residual feed intake. The RXFP4 gene encodes a receptor for an orexigenic peptide, insulin-like peptide 5 produced by intestinal $\mathrm{L}$ cells, which is expressed by enteric neurons. Olfactory receptors are critical for transmitting the effects of odorants, contributing to the sense of smell, and have been implicated in participating in appetite regulation.
\end{abstract}

Conclusions: Our results identify CNV for genomic evaluation in Holstein cattle, and provide candidate genes, such as RXFP4, contributing to variation in feed efficiency and feed intake-related traits. These results indicate potential novel targets for manipulating feed intake-related traits of livestock.

Keywords: Copy number variation (CNV), Dairy cow, Genome-wide association study, Feed intake, Feed efficiency

\section{Background}

Apart from single nucleotide polymorphism (SNP), copy number variation $(\mathrm{CNV})$ is another type of genetic marker that potentially affects animal phenotype [1]. These CNV consist of variation of genome sequences ranging from 50 to 5 million base pairs [2]. Compared to SNP, CNV show more drastic effects on gene expression and function, such as altering gene dosage, disrupting coding sequence, or perturbing long-range gene regulation [3]. However, until

\footnotetext{
* Correspondence: george.liu@ars.usda.gov

${ }^{1}$ Animal Genomics and Improvement Laboratory, BARC, USDA-ARS, 10300

Baltimore Avenue, Bldg. 306, BARC-East, Beltsville, MD 20705, USA

Full list of author information is available at the end of the article
}

recently, the effective use of $\mathrm{CNV}$ as genetic markers for association with diseases and economic phenotypes has been impaired by difficulties in accurately detecting $\mathrm{CNV}$ and their boundaries, which vary among individuals $[4,5]$. To date, especially within the livestock genomics field, most studies have focused exclusively on CNV discovery, and only a few studies and software have attempted to use comparative genomics methods to detect possible phenotype-related $\mathrm{CNV}$ in livestock using genome-wide association study (GWAS) [5-7].

Numerous methods have been used to detect CNV, ranging from traditional cytogenetic approaches, such as karyotyping and fluorescence in situ hybridization, to 
genome-wide in silico CNV prediction [8, 9]. Substantial improvements have been made recently to the accuracy and throughput of CNV discovery. For example, the application of microarray and next-generation sequencing technologies makes it possible to compare $\mathrm{CNV}$ among populations at a whole-genome scale, and sequencing methods offer opportunities to identify CNV in highly complex repetitive regions of the genome $[10,11]$. However, neither sequencing methods are economical or widely used for large population analysis, including GWAS. Thus, the use of SNP chips is now commonplace for SNP-based GWAS studies, including studies of large populations of livestock. As a result, software packages have been developed and published for CNV prediction from SNP chip data, such as the SNP \& Variation Suite (SVS; Golden Helix, Bozeman, MT), which facilitate a low-cost approach to detect common $\mathrm{CNV}$ within a population for GWAS analysis [5, 6].

Because Holsteins are the largest milk-producing dairy breed worldwide, GWAS examining reproduction, growth, milk production, and disease resistance traits among dairy cattle have been performed often in the Holstein breed. In these studies, SNP markers were used almost exclusively, whereas studies using CNV markers for GWAS are rare. For instance, Xu et al. [6] characterized $34 \mathrm{CNV}$ significantly associated with milk production traits using a CNV inferred from the BovineSNP50 array (Illumina, San Diego, CA), and Durán Aguilar et al. [12] applied CNV-based GWAS for milk somatic score using the BovineHD Genotyping BeadChip (Illumina) containing over $777 \mathrm{~K} \mathrm{SNP}[6,12]$. In a third study of Spanish Holsteins, 90 segregating CNV were identified from BovineSNP50 array hybridization signals and studied for their association with 7 production and conformation traits [7]. For other cattle breeds and livestock species, such as beef cattle and pigs, several GWAS have been conducted using CNV $[5,13]$.

Here, we applied CNV detection and GWAS analysis for 10 important production-related phenotypes of dairy cattle, including those related to feed intake, milk composition, female fertility, and productive life, as well as net merit. These studies were conducted using genotypes generated with the BovineHD Genotyping BeadChip (Illumina) from 528 Holstein cows. We identified $191 \mathrm{CNV}$ based on size and frequency, and found 57 $\mathrm{CNV}$ associated with at least one of those phenotypes.

\section{Methods}

\section{Animals}

Cows $(n=528)$ used in the study were registered Holsteins from the Beltsville Agricultural Research Center Dairy Herd located in Beltsville, MD. Genotyping was performed using high-quality genomic DNA extracted from white blood cells or hair follicles of cows using Gentra Puregene DNA extraction kits (Qiagen, Valencia, CA) and the BovineHD Genotyping BeadChip (Illumina).

\section{Phenotypes and PTA calculations}

Phenotypes were available for analysis from 473 of the genotyped cows. Measurements for estimation of DMI and RFI were performed as described previously [14]. Production traits of cows were collected by Dairy One Cooperative Inc. (Ithaca, NY) using ICAR-approved methods and quality certification standards administered by the Council on Dairy Cattle Breeding (Bowie, MD). Conventional PTA for RFI and DMI were calculated as described in Lu et al. [15], and PTA for milk fat percentage, milk protein percentage, cow conception rate, heifer conception rate, daughter pregnancy rate, somatic cell score, net merit, and productive life were calculated as described in VanRaden and Wiggans [16]. De-regressed PTA (dPTA) were calculated according to the formula: $\mathrm{dPTA}=$ PTA / reliability as described by Garrick et al. [17] and used as the phenotype for further CNV GWAS, similar to $\mathrm{Xu}$ et al. [6]. Although matrix deregression based on pedigree structure is expected to more accurately remove the contributions of other relatives to the final deregressed evaluation [18], we used the simpler removal procedure.

\section{CNV segmentation and genotyping}

The SNP genotypes generated by the Illumina BovineHD Genotyping BeadChip assay with a call rate $>90 \%$ were used to detect the common CNV shared among Holstein cows using methods previously described by Zhou et al. [5]. Specifically, the DSF file was exported from GenomeStudio Software and the Log R Ratios (LRR) were imported into SVS 8.3.0 ([19]; Golden Helix Inc.). A total of 735,293 SNP were successfully mapped onto the 29 autosomes of the Bos taurus genome assembly UMD 3.1 [20]. The default GC correction file in the SVS software was used to correct the LRR waviness caused by the GC content. The multivariate method was used to define the CNV segments, employing the SVS default settings which are based on the expected CNV density (count and length) within a genome, as follows: (1) a maximum of 20 segments per $10 \mathrm{~K}$ markers; (2) at least 3 markers per segment; and (3) a maximum pair-wise segment $P$-value of 0.005 . The 3 states with a comparatively strict threshold (segment mean \pm 0.4 ) defined the $\mathrm{CNV}$ as 3 types of events (loss [-1], neutral [0], or gain $[+1])$ across all of the samples.

\section{PCA-corrected association testing}

Multiple linear regression in an additive genetic model was used to identify the CNV significantly associated 
with 10 traits individually. Both FDR and 10,000 permutations were performed to correct for multiple testing. The model was as follows:

$$
y_{i}=\sum_{j=1}^{m} x_{i j} \beta_{j}+e_{i}
$$

where $y_{i}$ is the dPTA of the $i^{\text {th }}$ individual, $x_{i j}$ is the CNV genotype of the $i^{\text {th }}$ individual (gain, loss, and neutral as represented by $1,-1$ and 0 ), $\beta_{j}$ is the CNV effect, $m$ is the number of $\mathrm{CNV}$, and $e_{i}$ is the residual. To correct for batch effects/stratification, the Principal Component Analysis (PCA) option was selected, wherein the SVS software identified the optimum number of components based on a range from 1 to 20 . See Golden Helix SNP \& Variation Suite v8.3.0 Manual [19] for additional details on methodology. Significant $\mathrm{CNV}$ were identified after false discovery rate (FDR) correction $(P<0.05)[6]$.

\section{Evaluation of QTL and genetic overlap of CNV}

Gene annotations were downloaded from the Ensembl database [21] and QTL were downloaded from the animal QTL database [22] (based on the UMD3.1 bovine reference genome assembly). The overlaps between $\mathrm{CNV}$ and genes or QTL were detected using R3.3.3 GenomicRanges [23]. To detect potential genes affected by significant $\mathrm{CNV}$, we define the 'overlap' as more than $1 \mathrm{bp}$ in common between the CNV region and the genomic region (including the $5-\mathrm{Kb}$ flanking regions both up- and downstream) of a given gene transcript. Because of overly large confidence intervals (reported by the Animal QTLdb) for some QTL, we filtered out the QTL with confidence intervals $>30 \mathrm{Mb}$ and used a strict threshold to define the overlap as at least $50 \%$ of the CNV length being covered by the QTL.

\section{CNV validation by $\mathrm{qPCR}$}

For each CNV selected for validation, the Illumina Bovine HD SNP chip probe sequences corresponding to SNP within the CNV region of interest were identified. The 400-bp genomic DNA sequence surrounding each targeted SNP (i.e., \pm 200 bp of the SNP) was selected as the template sequence for PCR primer design using the NCBI Primer-BLAST primer designing tool [24]. The desired PCR product size was specified as 100 to $300 \mathrm{bp}$ and the best primer pair was selected from the output. Primer information can be found in Additional file 2: Table S1. Reactions $(25 \mu \mathrm{L})$ were performed in triplicate using SsoAdvanced Universal SYBR Green Supermix (Bio-Rad, Hercules, CA), 5 ng genomic DNA, and $400 \mathrm{n} M$ each primer on the Bio-Rad CFX96 Touch Real-Time PCR Detection System. Amplification consisted of $95{ }^{\circ} \mathrm{C}$ for $1 \mathrm{~min}$, followed by 45 cycles of $95{ }^{\circ} \mathrm{C}$ for $10 \mathrm{~s}, 58.2{ }^{\circ} \mathrm{C}$ for $10 \mathrm{~s}$, and $72{ }^{\circ} \mathrm{C}$ for $30 \mathrm{~s}$. Amplification of the expected product size was confirmed by electrophoresis on a $2 \%$ agarose gel. Melting curve analysis following the final PCR amplification step was used to confirm presence of a single amplicon. Efficiency of PCR amplification for each target was determined from a 4point standard curve using 1.5 to $24 \mathrm{ng}$ of genomic DNA as template, and ranged from 99.3 to $107.4 \%$. Linearity of all standard curves exceeded 0.995. The BTF3 gene and a common DNA sample from Hereford cow, L1 Dominette 01449 were used as references for all qPCR experiments. The $2^{-\Delta \Delta C}$ method [25] was employed to analyze qPCR results wherein we defined 0 to 1.5 copies as a copy loss $(-1),>1.5$ to 3 copies as neutral $(0)$, and $>3$ copies as gain $(+1)$.

\section{Phenotype correlation analysis}

Ten diverse phenotypes of RFI, DMI, milk fat percentage, milk protein percentage, somatic cell score, cow conception rate, heifer conception rate, daughter pregnancy rate, net merit, and productive life for $473 \mathrm{Hol}-$ stein cows were selected for correlation analysis to understand their relationships to one another. Pearson product-moment correlations were computed between pairs of APTA for all of the 10 phenotypes.

\section{Results and discussion \\ CNV segments and genotyping}

We detected 454 CNV from 528 Holstein cows where the $\mathrm{CNV}$ were characterized as 3 types $(-1,0$, and +1$)$. We filtered out likely false CNV using the strict threshold criteria of length $\leq 1 \mathrm{Mb}$ and frequency $>0.5 \%$. The resulting 191 common $\mathrm{CNV}$ had lengths ranging from 727 to $897,251 \mathrm{bp}$ and frequencies ranging from 1.1 to 98.9\% (Additional file 2: Table S2; Fig. 1). These CNV comprised $0.64 \%(16.2 \mathrm{Mb})$ of the whole autosomal length and were distributed differently among autosomes. The chromosome with the highest percentage of CNV length (3.8\%) was Chr15. Bovine Chr12 was reported as having the highest percentage of $\mathrm{CNV}$ length in a previous CNV study [11], whereas in the current study, Chr12 ranked second highest for percentage of CNV length among all autosomes. We detected 164 genes that overlapped with the CNV (Additional file 2: Table S2). A total of 47 or 189 of our 191 CNV events were overlapped with Ben Sassi et al. and Xu et al. (Additional file 2: Table S2) [6, 7]. Gene ontology (GO) analysis results of genes overlapping with $\mathrm{CNV}$ are typically enriched in functions related to immunity or response to stimuli [26]. However, in our DAVID analysis using Fisher's exact test, the CNV were highly enriched in GO terms related to development and growth, such as: multicellular organismal process, developmental process, and 


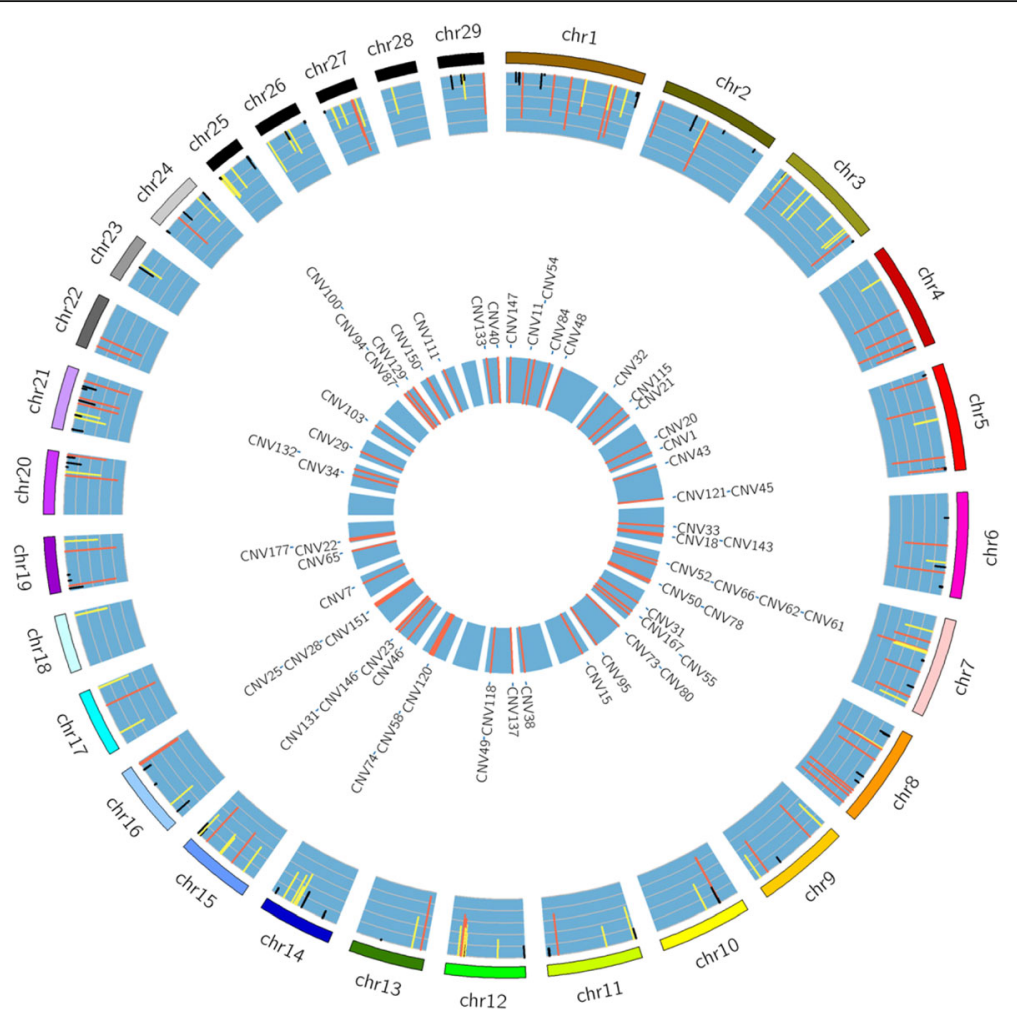

Fig. 1 Distribution of CNV and significantly associated CNV on each Bos taurus autosome. Outer circle: distribution of 191 CNV where the height and color of histograms represent the variant frequency of each CNV (red, > 0.6; yellow, 0.3 to 0.6; and black, < 0.3); Inner circle: distribution of CNV significantly associated with at least 1 of the 10 production traits evaluated

multicellular organismal development (Additional file 2: Table S3).

\section{Phenotype correlation analysis}

Figure 2 shows results of the Pearson correlation analysis between each of the dPTA. As described previously $[27,28]$, the 10 dPTA generally showed moderate to strong correlation coefficients within each phenotypic category (i.e., feed efficiency and intake [RFI and DMI], milk composition and quality [fat percentage, protein percentage, and somatic cell score], fertility [cow conception rate, heifer conception rate, and daughter pregnancy rate], and composite evaluation [net merit and productive life]), but weaker correlation coefficients between different phenotypic categories. For example, RFI versus DMI was moderate $(r=0.60, P<0.0001)$, milk fat versus protein percentage was moderate to high $(r=0.75, P<0.0001)$, and the correlation coefficients of the 3 fertility traits were variable, ranging from 0.27 to 0.89 . Net merit and productive life were highly correlated $(r=0.78, P<0.0001)$ with one another, and both were moderately correlated with the fertility phenotypes. Somatic cell score showed weak correlation with other phenotypes, such as productive life $(r=-0.33, P<0.0001)$ and net merit $(r=-0.26, \quad P<0.0001)$, and most coefficients were negative.

\section{Significant CNV association analysis}

Before applying the GWAS analysis, we excluded $2 \mathrm{CNV}$ because they appeared in fewer than 5 individuals. The frequency of the remaining $189 \mathrm{CNV}$ among the 473 cows ranged from 1.3 to $98.9 \%$. In total, we detected 57 CNV associated after FDR correction $(P<0.05)$ with at least 1 of the 10 phenotypes (Additional file 2: Table S4; Fig. 3). These significant CNV were distributed among 25 autosomal chromosomes (Fig. 1), wherein no significant CNV were identified on Chr13, Chr20, Chr24, or Chr28. We did not observe strong bias due to the frequency of CNV present on a given chromosome on the number of significant $\mathrm{CNV}$ detected within that chromosome. Chr1 and Chr12 had the greatest CNV number for GWAS analysis, but based on CNV density (i.e., CNV count normalized by chromosome length), we did not find obvious enrichment of significant CNV on these 2 chromosomes. However, Chr7 was enriched with the greatest number $(n=6)$ of CNV significantly associated with the production traits of interest. The length of the 57 significant $\mathrm{CNV}$ ranged from 1.2 to $350 \mathrm{~Kb}$ and their frequencies ranged from 4.6 to $98.9 \%$. We selected 


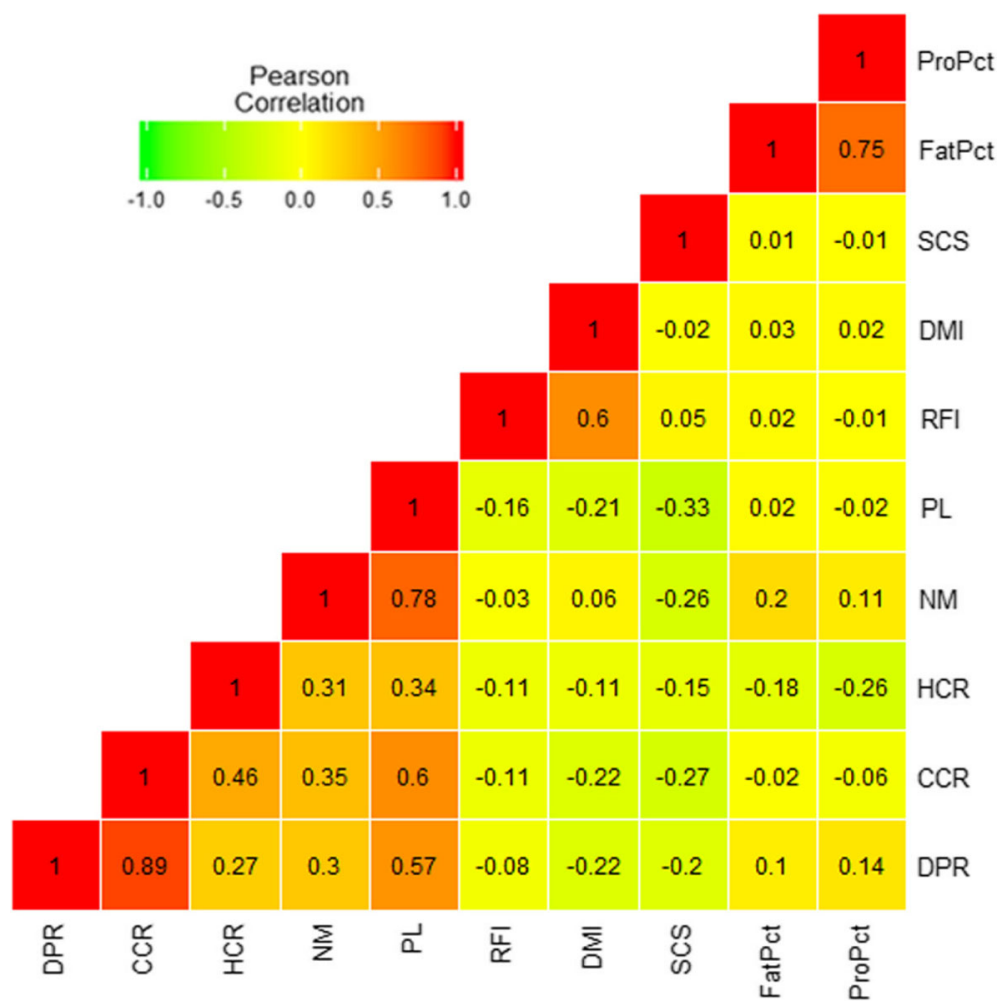

Fig. 2 Pair-wise Pearson correlation coefficients for 10 dairy production-related phenotypes of interest. DPR: daughter pregnancy rate; CCR: cow conception rate; HCR: heifer conception rate; NM: net merit; PL: productive life; RFI: residual feed intake; DMl: dry matter intake; SCS: somatic cell score; FatPct: fat percentage; and ProPct: protein percentage

7 significant $\mathrm{CNV}$ associated with multiple traits of interest or overlapping known genes or QTL for validation among 8 animals using qPCR. As most of the CNV (90\%) were deletions, all 7 selected CNV were deletions. For 3 out of 7 cases, the PCR amplicon for CNV validation was within genes: CNV33 $(\mathrm{RHOH}), \mathrm{CNV} 46$ (GRIK4), and CNV40 (AP2A2). The result showed 55.4\% of the 56 qPCR results were consistent with the in silico prediction (Additional file 2: Table S5), which is typically in the range of 60 to $70 \%$ concordance [29]. Inconsistencies between $\mathrm{qPCR}$ and in silico prediction may occur due to multiple reasons, such as complex sequence of the genome, artificial assembly, probe bias, primer design, DNA quality, or other factors during array hybridization or PCR amplification.

As expected, the significant $\mathrm{CNV}$ were associated with phenotypes of different categories, with the number of significant $\mathrm{CNV}$ associated with a given trait ranging from 1 (e.g., dry matter intake) to 19 (e.g., productive life; Additional file 2: Table S4). Phenotypes with higher correlation are more likely to share significant CNV. For example, we found 6 significant CNV (CNV11, CNV23, CNV29, CNV50, CNV121, CNV137) associated with both cow conception rate and daughter pregnancy rate; traits which were highly correlated. Similarly, another highly correlated phenotypic pair of net merit and productive life shared 10 significant CNV (CNV20, CNV40, CNV43, CNV45, CNV55, CNV62, CNV80, CNV94, CNV118, CNV121), whereas RFI and DMI shared only 1 significant CNV (CNV1). The most dissimilar phenotype in the correlation analysis, somatic cell score, was found to share no significant $\mathrm{CNV}$ with any of the other phenotypes evaluated. The relationships between phenotypes will help to better define potentially related markers as significant CNV appearing with both or all highly correlated phenotypes will be more reliable than those $\mathrm{CNV}$ associated with only 1 phenotype.

\section{Genes and QTL overlapping with significant CNV}

We detected 54 Ensembl gene ID overlapping with 20 significant CNV (Additional file 2: Table S4). Among them, CNV150 (Chr26: 25,719,640-26,013,587) overlapped with the greatest number of genes $(n=13)$ with all genes located inside the $\mathrm{CNV}$ region. There were 11 significant CNV (CNV147, CNV32, CNV33, CNV18, CNV66, CNV146, CNV151, CNV177, CNV34, CNV150, and $\mathrm{CNV40)}$ that overlapped with genes previously reported in cattle, or other species, to harbor deletions or duplications, and several others overlapping known gene regions. For instance, ELF3 was identified as a candidate 


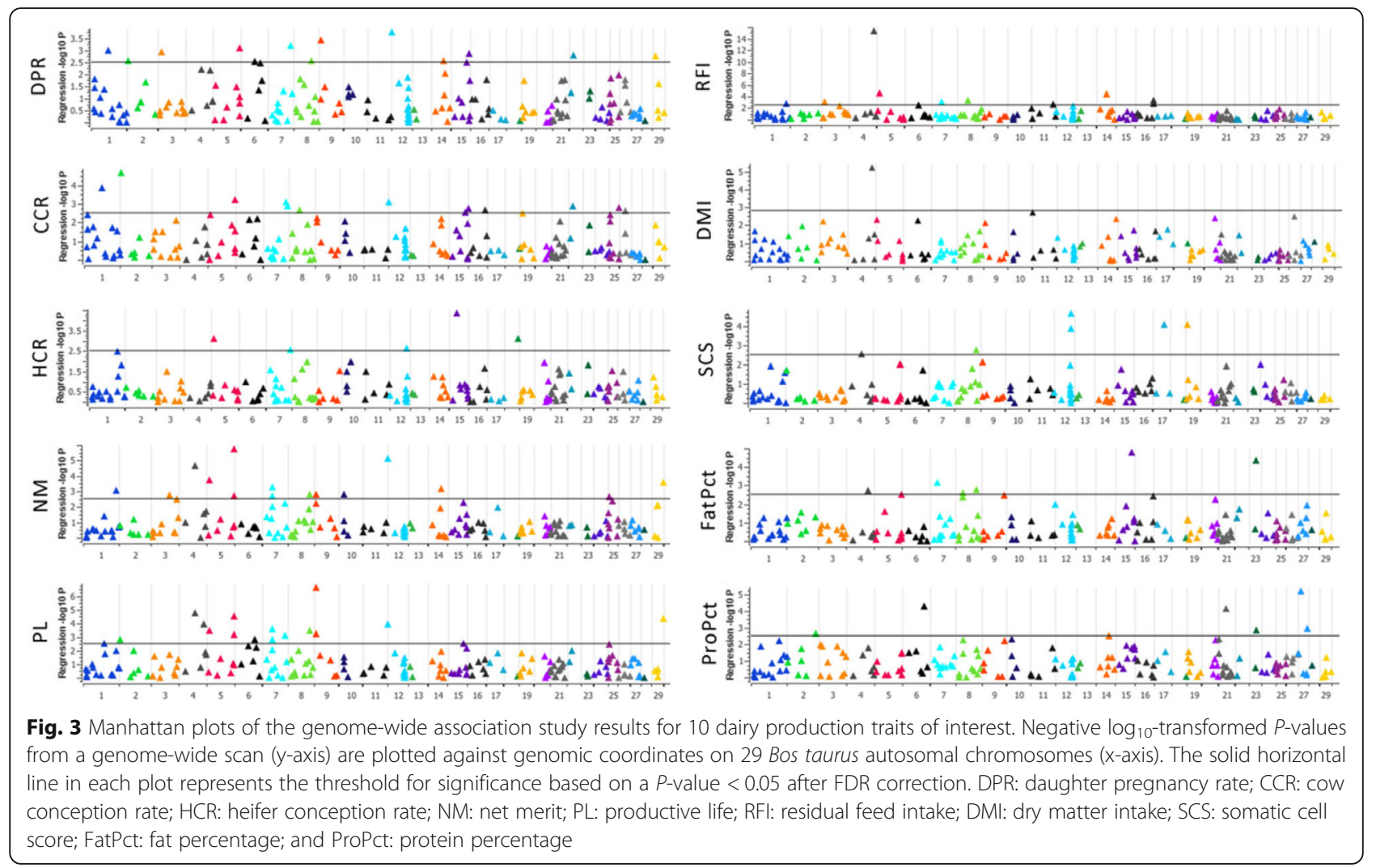

gene for somatic cell score in a previous CNV-based GWAS study [12]. In the present study, CNV151 was associated with cow conception rate and is overlapping with the ELF3 gene. The product of the ELF3 gene is a transcription factor that functions in inflammation and epithelial cell differentiation, and may be involved in mammary gland development and involution [30], supporting its potential link to somatic cell score. How its function may relate to cow conception rate is not known.

Over half of the significant CNV were overlapping with at least 1 QTL (Additional file 2: Table S4), of which some were for traits that support our GWAS results. For example, CNV46 was significantly associated with heifer conception rate and it overlaps with a QTL for the circulating level of the reproductive hormone, LH. In addition, CNV23 was significantly associated with cow conception rate and daughter pregnancy rate, and it overlaps with a QTL for first-service conception rate. Lastly, CNV66 was significantly associated with RFI and overlaps with a QTL for average daily weight gain, and CNV7 was associated with somatic cell score and overlaps with multiple QTL for somatic cell score and clinical mastitis. Thus, further evaluation of these $\mathrm{CNV}$ is warranted in additional cattle populations for their association with these, or similar, production traits.

\section{Characterization of shared significant CNV for phenotypes} related to feed efficiency and intake

We focused our study on the 2 phenotypes of RFI and DMI related to feed efficiency and intake. There were 10 and $1 \mathrm{CNV}$ significantly associated with RFI and DMI, respectively (Table 1 ). The CNV shared by RFI and DMI was CNV1 (Chr4: 108,225,979-108,252,635; $P \leq 0.001$ ), which overlaps predicted olfactory receptor gene OR2A2 (LOC787786). However, only 6 cows were typed for CNV1 as neutral (0), while the remaining 467 cows were all typed as loss $(-1)$. Although the 6 neutral cows had a greater mean DPTA for both RFI and DMI, we identified some cows possessing the CNV1 loss type that had equal or even higher dPTA than the mean DPTA of the CNV1 neutral types (Additional file 1: Figure S1). Thus, particular attention must be given to significant CNV occurring at very low or high frequency when considering them as candidate markers for use in animal breeding.

The CNV32 (Chr3: 14,876,353-14,882,132) associated with RFI also only showed loss and neutral types in our population, with a loss frequency of $81.0 \%$. It is located $3.7 \mathrm{~Kb}$ upstream of the relaxin/insulin like family peptide receptor 4 (RXFP4; Chr3:14,871,054-14,872,623; strand: -) gene transcription start site, which is potentially affecting RXFP4 gene expression by altering its promoter region. Of interest, the ligand for RXFP4 is 
Table 1 Significant CNV from genome-wide association analysis for RFI and DMI traits among Holstein cattle

\begin{tabular}{|c|c|c|c|c|c|c|c|c|}
\hline \multirow[b]{2}{*}{ CNV } & \multirow[b]{2}{*}{ Chromosome } & \multirow[b]{2}{*}{ Start (bp) } & \multirow[b]{2}{*}{ End (bp) } & \multicolumn{2}{|c|}{$\begin{array}{l}P \text {-value after } \\
\text { FDR correction }\end{array}$} & \multicolumn{3}{|l|}{ Overlapped Ensemble } \\
\hline & & & & $\mathrm{DMl}$ & RFI & Gene ID & Gene Symbol & Overlapped QTL \\
\hline CNV1 & 4 & $108,225,979$ & $108,252,635$ & 0.0012 & 0.0000 & & $\begin{array}{l}\text { LOC787786 } \\
\text { (OR2A2) }\end{array}$ & \\
\hline CNV25 & 16 & $79,851,676$ & $79,853,884$ & 0.2802 & 0.0236 & & & \\
\hline CNV28 & 16 & $78,028,784$ & $78,035,505$ & 0.9670 & 0.0367 & ENSBTAG000000002291 & ZBTB41 & \\
\hline CNV31 & 8 & $44,934,828$ & $44,944,671$ & 0.3442 & 0.0201 & & LOC101909075 & \\
\hline CNV32 & 3 & $14,876,353$ & $14,882,132$ & 0.1561 & 0.0259 & ENSBTAG00000046885 & RXFP4 & \\
\hline CNV38 & 11 & $93,188,055$ & $93,190,178$ & 0.6321 & 0.0391 & & & Stearic acid content \\
\hline CNV43 & 5 & $9,756,491$ & $9,757,695$ & 0.4402 & 0.0027 & & & $\begin{array}{l}\text { Infectious bovine keratoconjunctivitis } \\
\text { susceptibility, Cold tolerance, Calving } \\
\text { ease (maternal), Male fertility, Gestation } \\
\text { length, Milk alpha-lactalbumin \%, } \\
\text { Retained placenta (DYD), Milk protein \% }\end{array}$ \\
\hline \multirow[t]{2}{*}{ CNV66 } & 7 & $42,745,346$ & $42,788,788$ & 0.4395 & 0.0274 & ENSBTAG00000046318 & $\begin{array}{l}\text { LOC787816 } \\
\text { (OR2T12) }\end{array}$ & Cold tolerance \\
\hline & & & & & & ENSBTAG00000007557 & OR2AK2 & Average daily gain \\
\hline CNV84 & 1 & $143,922,352$ & $143,945,292$ & 0.7750 & 0.0357 & & & \\
\hline CNV120 & 14 & $44,862,013$ & $44,868,983$ & 0.3398 & 0.0025 & & & Subcutaneous fat \\
\hline
\end{tabular}

Note: See Additional file 2: Table S4 for additional details. $P$-values $<0.05$ are indicated in bold font

insulin-like peptide 5 (Insl5), which is produced by intestinal $\mathrm{L}$ cells in response to a reduction in feed intake, stimulating appetite [31]. In humans, RXFP4 was found to be significantly associated with obesity and body mass index [32]. Studies also reported that Insl5-RXFP4 signaling plays a role in glucose metabolism [33]. Thus, CNV32 may affect feed efficiency of cattle through an RXFP4-mediated pathway and provides an interesting candidate gene for further study in cattle.

Another significant CNV associated with RFI, CNV66 (Chr7:42,745,346-42,788,788), showed both loss and gain types with a total frequency of $54.6 \%$ within our Holstein population. Similar to CNV1, 2 olfactory receptor genes of predicted OR2T12 (LOC787816) and OR2AK2 are located inside CNV66. Olfactory receptors may influence feeding behavior, such as food preference and feed intake [34]. The variation of olfactory receptor gene copy numbers, theoretically, could affect their expression levels and impact RFI and DMI of Holstein cattle. These olfactory receptor genes serve as additional candidates for further study in the regulation of feed efficiency and feed intake of dairy cattle.

\section{Conclusions}

Our previous study showed that $\sim 25 \%$ of CNV did not have a significant association with SNP; thus the effects of these CNV probably were not captured by tag SNP [6]. In this study, we performed a CNV-based GWAS for 10 important production traits and detected $57 \mathrm{CNV}$ significantly associated with at least one of these production traits. Of particular interest regarding feed intake- related phenotypes, we detected $2 \mathrm{CNV}$ associated with RFI located within RXFP4, encoding a G-protein coupled receptor thought to play a role in regulation of appetite and metabolism [31, 35], and 2 olfactory receptor gene regions, respectively. We also identified a single CNV within predicted OR2A2 strongly associated with both RFI and DMI. Our results identify CNV for genomic evaluation in Holstein cattle, and provide candidate genes contributing to variation in feed efficiency and feed intake-related traits.

\section{Additional files}

Additional file 1: Figure S1. Box plot for dPTA distribution of the 2 feed intake-related phenotypes of RFI and DMI. (PDF 90 kb)

Additional file 2: Table S1. Primer information for CNV validation. Table S2. Characteristics of CNV and their overlapped genes. Table S3. Gene ontology (GO) of genes that overlap with CNV. Table S4. CNVbased GWAS results for the 10 phenotypes of interest among Holstein cattle. Table S5. qPCR validation of the significant CNV. (XLSX 96 kb)

\section{Acknowledgements}

The authors acknowledge the excellent technical support of Mary Bowman, Christina Evock-Clover, Donald Carbaugh, Jonathan Leith, Mike Kemp, and Research Support Services staff at the USDA-ARS (Beltsville, MD) who contributed to this project. The Agriculture and Food Research Initiative Competitive Grant no. 2011-68004-30340 enabled calculation of traditional PTA for RFI and DMI used in the study and provided partial support for cow genotyping. Mention of trade names or commercial products in this article is solely for the purpose of providing specific information and does not imply recommendation or endorsement by the US Department of Agriculture. The USDA is an equal opportunity provider and employer. 


\section{Funding}

This work was supported by the U.S. Department of Agriculture, Agricultural Research Service Project numbers 8042-31320-077-00-D and 8042-31000-104-00-D, and in part by The Agriculture and Food Research Initiative Competitive Grant no. 2011-68004-30340.

\section{Availability of data and materials}

Genotyping data are available upon request from the corresponding author for research purposes and with permission of the ARS Technology Transfer Coordinator

\section{Authors' contributions}

$Y Z, G E L$, and EEC conceived and designed the experiments. $Y Z, G R W, Y L$, $R J T$, and HC performed in silico prediction and/or computational analyses. SGS collected and managed SNP array data. EEC collected samples and feed-related phenotypes, and performed $\mathrm{QPCR}$ confirmation. $Y Z$, EEC, and GEL wrote the manuscript. All authors read and approved the final manuscript.

\section{Ethics approval}

All procedures involving animals were approved by the Beltsville Agricultural Research Center's Institutional Animal Care and Use Committee, protocol numbers 07-016, 10-013, 13-018, and 16-004.

\section{Competing interests}

The authors declare that they have no competing interests.

\section{Publisher's Note}

Springer Nature remains neutral with regard to jurisdictional claims in published maps and institutional affiliations.

\section{Author details}

${ }^{1}$ Animal Genomics and Improvement Laboratory, BARC, USDA-ARS, 10300 Baltimore Avenue, Bldg. 306, BARC-East, Beltsville, MD 20705, USA. ${ }^{2}$ Shaanxi Key Laboratory of Agricultural Molecular Biology, College of Animal Science and Technology, Northwest A\&F University, Yangling 712100, Shaanxi, China. ${ }^{3}$ Key Laboratory of Agricultural Animal Genetics, Breeding and Reproduction, Education Ministry of China, Huazhong Agricultural University, Wuhan 430070, Hubei, China. ${ }^{4}$ Department of Animal Science, Michigan State University, East Lansing, MI 48824, USA.

\section{Received: 17 May 2017 Accepted: 18 April 2018}

\section{Published online: 02 May 2018}

\section{References}

1. Bickhart DM, Liu GE. The challenges and importance of structural variation detection in livestock. Front Genet. 2014;5:37.

2. Mills RE, Walter K, Stewart C, Handsaker RE, Chen K, Alkan C, et al. Mapping copy number variation by population-scale genome sequencing. Nature. 2011:470:59-65.

3. Zhang F, Gu W, Hurles ME, Lupski JR. Copy number variation in human health, disease, and evolution. Annu Rev Genomics Hum Genet. 2009:10:451-81.

4. Pinto D, Darvishi K, Shi X, Rajan D, Rigler D, Fitzgerald T, et al. Comprehensive assessment of array-based platforms and calling algorithms for detection of copy number variants. Nat Biotechnol. 2011;29:512-20.

5. Zhou Y, Utsunomiya YT, Xu L, Hay EHA, Bickhart DM, Alexandre PA, et al. Genome-wide CNV analysis reveals variants associated with growth traits in Bos indicus. BMC Genomics. 2016:17:419.

6. Xu L, Cole JB, Bickhart DM, Hou Y, Song J, VanRaden PM, et al. Genome wide CNV analysis reveals additional variants associated with milk production traits in Holsteins. BMC Genomics. 2014;15:683.

7. Ben Sassi N, González-Recio Ó, de Paz-del Río R, Rodríguez-Ramilo ST, Fernández Al. Associated effects of copy number variants on economically important traits in Spanish Holstein dairy cattle. J Dairy Sci. 2016;99:6371-80.

8. Buysse K, Delle Chiaie B, Van Coster R, Loeys B, De Paepe A, Mortier G, et al. Challenges for CNV interpretation in clinical molecular karyotyping: lessons learned from a 1001 sample experience. Eur J Med Genet. 2009:52:398-403.

9. Zhao M, Wang Q, Wang Q, Jia P, Zhao Z. Computational tools for copy number variation (CNV) detection using next-generation sequencing data: features and perspectives. BMC Bioinformatics. 2013;14(Suppl 11):S1.
10. Bickhart DM, Xu L, Hutchison JL, Cole JB, Null DJ, Schroeder SG, et al. Diversity and population-genetic properties of copy number variations and multicopy genes in cattle. DNA Res Int J Rapid Publ Rep Genes Genomes. 2016;23:253-62.

11. Zhou Y, Utsunomiya YT, Xu L, Hay EHA, Bickhart DM, Sonstegard TS, et al. Comparative analyses across cattle genders and breeds reveal the pitfalls caused by false positive and lineage-differential copy number variations. Sci Rep. 2016;6:29219.

12. Durán Aguilar M, Román Ponce SI, Ruiz López FJ, González Padilla E, Vásquez Peláez CG, Bagnato A, et al. Genome-wide association study for milk somatic cell score in Holstein cattle using copy number variation as markers. J Anim Breed Genet Z Tierzuchtung Zuchtungsbiologie. 2017;134:49-59.

13. Wang $L, X u L$, Liu X, Zhang $T$, Li N, Hay EH, et al. Copy number variationbased genome wide association study reveals additional variants contributing to meat quality in swine. Sci Rep. 2015:5:12535.

14. Connor EE, Hutchison JL, Norman HD, Olson KM, Van Tassell CP, Leith JM, et al. Use of residual feed intake in Holsteins during early lactation shows potential to improve feed efficiency through genetic selection. J Anim Sci. 2013:91:3978-88.

15. Lu Y, Vandehaar MJ, Spurlock DM, Weigel KA, Armentano LE, Staples CR, et al. An alternative approach to modeling genetic merit of feed efficiency in dairy cattle. J Dairy Sci. 2015;98:6535-51.

16. VanRaden PM, Wiggans GR. Derivation, calculation, and use of national animal model information. J Dairy Sci. 1991;74:2737-46.

17. Garrick DJ, Taylor JF, Fernando RL. Deregressing estimated breeding values and weighting information for genomic regression analyses. Genet Sel Evol GSE. 2009;41:55.

18. Calus MPL, Vandenplas J, Ten Napel J, Veerkamp RF. Validation of simultaneous deregression of cow and bull breeding values and derivation of appropriate weights. J Dairy Sci. 2016:99:6403-19.

19. SNP \& Variation Suite Manual — SNP \& Variation Suite v8.3.0 Manual. http:// doc.goldenhelix.com/SVS/8.3.0/svs_index.html. Accessed 11 May 2017.

20. The Bovine Genome Database. University of Missouri, Columbia. UMD 3.1 Downloads (Latest) | BovineGenome.org. http://www.bovinegenome.org/ ?q=node/61. Accessed 8 June 2016.

21. Ensembl genome browser 88. http://useast.ensembl.org/index.html. Accessed 1 July 2016.

22. Animal QTL Database. http://www.animalgenome.org/cgi-bin/QTLdb/index. Accessed 6 July 2016.

23. R Core Team. R: A language and environment for statistical computing. Vienna: R Foundation for Statistical Computing; 2013. http://www.R-project. org/. Accessed 6 July 2016.

24. Primer designing tool. https://www.ncbi.nlm.nih.gov/tools/primer-blast/. Accessed 20 Aug 2016.

25. Livak KJ, Schmittgen TD. Analysis of relative gene expression data using real-time quantitative $P C R$ and the 2(-Delta Delta $C(T))$ method. Methods San Diego Calif. 2001;25:402-8.

26. Liu GE, Hou Y, Zhu B, Cardone MF, Jiang L, Cellamare A, et al. Analysis of copy number variations among diverse cattle breeds. Genome Res. 2010;20:693-703.

27. Arthur PF, Archer JA, Johnston DJ, Herd RM, Richardson EC, Parnell PF. Genetic and phenotypic variance and covariance components for feed intake, feed efficiency, and other postweaning traits in Angus cattle. J Anim Sci. 2001:79:2805-11.

28. Brotherstone S. Genetic and phenotypic correlations between linear type traits and production traits in Holstein-Friesian dairy cattle. Anim Prod. 1994: 59:183-7.

29. Hou Y, Liu GE, Bickhart DM, Matukumalli LK, Li C, Song J, et al. Genomic regions showing copy number variations associate with resistance or susceptibility to gastrointestinal nematodes in Angus cattle. Funct Integr Genomics. 2012;12:81-92.

30. Oliver JR, Kushwah R, Hu J. Multiple roles of the epithelium-specific ETS transcription factor, ESE-1, in development and disease. Lab Investig J Tech Methods Pathol. 2012;92:320-30.

31. Grosse J, Heffron H, Burling K, Akhter Hossain M, Habib AM, Rogers GJ, et al. Insulin-like peptide 5 is an orexigenic gastrointestinal hormone. Proc Natl Acad Sci U S A. 2014;111:11133-8.

32. Kao ACC, Müller DJ. Genetics of antipsychotic-induced weight gain: update and current perspectives. Pharmacogenomics. 2013;14:2067-83.

33. Yegorov S, Bogerd J, Good SV. The relaxin family peptide receptors and their ligands: new developments and paradigms in the evolution from jawless fish to mammals. Gen Comp Endocrinol. 2014;209:93-105. 
34. Soria-Gomez E, Bellocchio L, Marsicano G. New insights on food intake control by olfactory processes: the emerging role of the endocannabinoid system. Mol Cell Endocrinol. 2014;397:59-66.

35. Ang SY, Hutchinson DS, Evans BA, Hossain MA, Patil N, Bathgate RAD, et al.

The actions of relaxin family peptides on signal transduction pathways

activated by the relaxin family peptide receptor RXFP4. Naunyn

Schmiedeberg's Arch Pharmacol. 2017;390:105-11.

Ready to submit your research? Choose BMC and benefit from:

- fast, convenient online submission

- thorough peer review by experienced researchers in your field

- rapid publication on acceptance

- support for research data, including large and complex data types

- gold Open Access which fosters wider collaboration and increased citations

- maximum visibility for your research: over $100 \mathrm{M}$ website views per year 